\title{
A LINK IN THE CHAIN OF SURVIVAL: NSW AMBULANCE RESPONSE TO CHEST PAIN
}

Shing-Chung Simon Fung, Glenn Close

Cardiovascular Disease Unit

NSW Health Department

John Hall

NSW Ambulance Service

$T^{\mathrm{H}}$ his report reviews the effectiveness of the ambulance transport component of the system for emergency response to acute chest pain in NSW, using routinely collected data.

Chest pain is an important symptom of coronary ischaemia and is the key to early recognition of acute coronary ischaemia (acute myocardial infarction and unstable angina) by the general community. Although the ratio of cases of confirmed acute myocardial infarct (AMI) to noncoronary chest pain may be low w $^{1,2,3}$, an effective system for management of those with chest pain will be the basis for effective delivery of definitive care of acute ischaemia.

The "Chain of Survival" model ${ }^{4}$ of emergency care emphasises the importance of:

$\square$
$\square$
$\square$ rapid public access to emergency care; training in cardio-pulmonary resuscitation techniques;

early defibrillation; and

prompt access to advanced cardiac life support.

To this should be added:

맘 early thrombolysis; and prompt access to advanced management and monitoring.

Effective delivery of care according to this model requires attention to issues such as symptom recognition in the community, pre-hospital care (including cardio-pulmonary resuscitation and defibrillation), response time by ambulance services, diagnosis and emergency treatment.

\section{METHODS}

The NSW Ambulance Service has a written protocol for the management of chest pain. When the Ambulance Service responds to a call for assistance, patient demographic details, clinical conditions, transportation mode and timing and any pre-hospital care given are recorded in the "ambulance patient report form". These data were entered in a database.

Data were analysed for "casualty calls" (i.e. not booked transport), with a complaint of chest pain, for the period January to June 1994.

Hospital separations for AMI (defined by the ICD.9.CM rubric 410) from the NSW Health Department 1993-94 Inpatient Statistics Collection (ISC) were also analysed to assess utilisation of ambulance services in relation to a discharge diagnosis of AMI.

\section{RESULTS}

There were 309,839 ambulance transportations in New South Wales during the study period (the first six months of 1994). Of these, 172,817 were casualty calls (56 per cent).

Of the 172,817 casualty calls, 10,743 (6.2 per cent) involved chest pain, although 751 ( 7.0 per cent) of these calls were cancelled, and a further 115 (1.1 per cent) were inter- hospital transfers or discharges from hospital to home (possibly miscoded as casualty calls). The remaining 9,877 ( 5.7 per cent) were confirmed casualty calls, managed by the Ambulance Service and transported to hospitals. Data for those calls formed the basis of our analysis.

\section{Chest pain protocol}

Of the 9,877 definite casualty chest pain cases transported by ambulance, 8,336 (84.4 per cent) were managed using the Ambulance Service's chest pain protocol. The rest were mainly managed under their basic protocol. The chest pain protocol recommends that glyceryl trinitrate be given if "myocardial ischaemia is suspected and systolic blood pressure is greater than $100 \mathrm{~mm} \mathrm{Hg}$ ", and that aspirin be given if coronary artery occlusion is suspected and there is no history of aspirin allergy.

Only about one in four ( 23.7 per cent) patients who were managed under the chest pain protocol were given aspirin, though nearly two-thirds (62.7 per cent) were given glyceryl trinitrate. About one in three ( 34 per cent) were given neither aspirin nor glyceryl trinitrate while in transit.

Time of call for ambulance assistance

There were no data recording the time from onset of chest pain. The ambulance record shows only the time of requesting ambulance assistance. These records showed a peak in requests for ambulance assistance for chest pain between $7 \mathrm{am}$ and noon. Another peak occurred between $6 \mathrm{pm}$ and $8 \mathrm{pm}$.

The NSW Ambulance Service received an average of 54 calls a day for chest pain. There was no substantial variation by day of the week, although Monday had the most cases on average (57), followed by Sunday (56). This pattern of variations was similar to the results of the 1982 Ambulance Inquiry ${ }^{5}$.

\section{Ambulance performance}

The time from call for assistance to ambulance team leaving the depot and the time from call for assistance to arrival at the scene (response time), are important indicators of service quality.

Overall, the time from calling the ambulance to arrival at hospital was less than 30 minutes in 31.3 per cent of cases, less than 45 minutes in 77.7 per cent and less than 60 minutes in 92.7 per cent of cases (Table 1).

The Ambulance Service is considering standards ${ }^{6}$ which have been developed and are used in the United Kingdom (UK) based on a metropolitan service. These standards have not been validated in NSW.

The NSW ambulance data show that, in about 80 per cent of cases, the ambulance left the depot within three minutes of receiving the request (Table 2). In about 37 per cent of cases in NSW, the ambulance arrived at the scene within seven minutes of receiving the call and in 84 per cent of cases, the ambulance arrived at the scene within 14 minutes.

Comparisons can be made between the NSW performance and the UK standards for urban and rural areas (Table 2). For example, in NSW urban areas the ambulance reached the scene within seven minutes of an emergency call in 32 per cent of cases, compared to the UK urban standard of 50 per cent. In NSW rural areas the corresponding proportion was 54 per cent within seven minutes, compared 
TABLE 1

PROPORTION (\%) OF AMBULANCE CASUALTY CALLS FOR CHEST PAIN WITHIN DEFINED CATEGORIES OF TIME FROM REQUEST FOR AMBBULANCE TO ARRIVAL AT HOSPITAL, JANUARY TO JULY 1994.

\begin{tabular}{|c|c|c|c|c|c|c|c|c|}
\hline Within & $5 \mathrm{~min}$ & Within $30 \mathrm{~min}$ & Within $45 \mathrm{~min}$ & Within $60 \mathrm{~min}$ & Within $75 \mathrm{~min}$ & Within 90 min & Within $105 \mathrm{~min}$ & Within $120 \mathrm{~min}$ \\
\hline Urban & 0.8 & 27.8 & 78.3 & 94.4 & 97.9 & 98.9 & 99.4 & 99.6 \\
\hline Rural & 2.5 & 42.9 & 75.8 & 87.0 & 93.4 & 96.6 & 98.1 & 98.8 \\
\hline NSW & 1.2 & 31.3 & 77.7 & 92.7 & 96.7 & 98.4 & 99.1 & 99.5 \\
\hline
\end{tabular}

\section{TABLE 2}

PROPORTION NSW AMBULANCE SERVICE CASUALTY TRANSPORTS FOR CHEST PAIN FALLING WITHIN UK URBAN/RURAL STANDARD TIMES FROM CALL TO LEAVING THE DEPOT AND ARRIVING AT THE SCENE, JANUARY TO JULY 1994.

\begin{tabular}{|c|c|c|c|c|c|c|}
\hline & \multicolumn{2}{|c|}{ From call to leaving depot } & \multicolumn{2}{|c|}{ From call to arrival at scene } & \multicolumn{2}{|c|}{ From call to arrival at scene } \\
\hline & $\begin{array}{r}\text { NSW } \\
\text { study } \\
\% \text { (time) }\end{array}$ & $\begin{array}{r}\text { UK } \\
\text { standard } \\
\% \text { (time) }\end{array}$ & $\begin{array}{r}\text { NSW } \\
\text { study } \\
\% \text { (time) }\end{array}$ & $\begin{array}{r}\text { UK } \\
\text { standard } \\
\% \text { (time) }\end{array}$ & $\begin{array}{r}\text { NSW } \\
\text { study } \\
\% \text { (time) }\end{array}$ & $\begin{array}{r}\text { UK } \\
\text { standard } \\
\% \text { (time) }\end{array}$ \\
\hline Urban & 83 (3 min) & 95 (3 min) & $32(7 \mathrm{~min})$ & $50(7 \mathrm{~min})$ & 85 (14 min) & 95 (14 min) \\
\hline Rural & 69 (3 min) & 95 (3 min) & $54(7 \mathrm{~min})$ & 50 (8 min) & $82(14 \mathrm{~min})$ & 95 (18 min) \\
\hline Total & 80 (3 min) & $\mathrm{n} / \mathrm{a}$ & 37 (7 min) & $n / a$ & 84 (14 min) & $\mathrm{n} / \mathrm{a}$ \\
\hline
\end{tabular}

\section{TABLE 3}

PROPORTION (\%) OF NSW AMBULANCE SERVICE CASUALTY CALLS FOR CHEST PAIN FALLING WITHIN SPECIFIED ACTIVATION AND RESPONSE TIMES, JANUARY TO JULY 1994.

\begin{tabular}{|c|c|c|c|}
\hline & $\begin{array}{l}\text { From call to leaving depot } \\
\text { (5 min) }\end{array}$ & $\begin{array}{l}\text { Response time } \\
\text { (10 } \mathrm{min})\end{array}$ & $\begin{array}{l}\text { Response time } \\
\text { (15 min) }\end{array}$ \\
\hline $\begin{array}{l}\text { Urban } \\
\text { Rural } \\
\text { NSW }\end{array}$ & $\begin{array}{l}94 \\
90 \\
93\end{array}$ & $\begin{array}{l}63 \\
72 \\
65\end{array}$ & $\begin{array}{l}88 \\
84 \\
87\end{array}$ \\
\hline
\end{tabular}

Data source: NSW Ambulance Service 1994 (HOIST), Epidemiology Branch, NSW Health Department.

to the UK rural standard of 50 per cent within eight minutes.

If the conventional five-minute intervals are used to measure performance, the ambulance data show that in 93 per cent of cases, the ambulance left the depot within five minutes of receiving the request. About 87 per cent of the time the ambulance arrived at the scene within 15 minutes, and about 96 per cent of the time within 30 minutes, of the time of receiving the request (Table 3 ).

There were some rural and urban differences in time from ambulance call to arrival at scene (Tables 1 and 2), with time from call to leaving depot being longer and response time shorter in rural than urban areas.

Hospitalisation: ambulance-transported AMI patients In 1993-94 there were 10,839 hospital separations in NSW where AMI was the principal diagnosis. Of these, 1,088 (10 per cent) had an ambulance number in their patient record, indicating they were brought in by an ambulance. The majority ( 90 per cent) of the 10,839 people with an AMI arrived at hospital using their own transportation arrangement.

\section{DISCUSSION}

These data show that, though there is room for improvement, the response times of the NSW Ambulance Service appear to be very good overall, even in relation to the UK standards. These are based on the UK experience and need to be validated in a local context.

Observed differences between rural and urban areas may be due, at least in part, to geographic conditions. It should be noted that, if the call is between midnight and 6am in rural areas there may be a slight delay as most officers are called in from home to attend the case.

A more significant issue may be the number of people who access the ambulance system at all. According to the 199394 NSW ISC, the ambulance services transported only 10 per cent of the AMI patients admitted to NSW hospitals. The recording of an ambulance number in the ISC almost certainly underestimates the number of people who arrive by ambulance. Nevertheless, even if this underestimation is of the order of 100 per cent, it seems that only about one in 


\section{NSW Ambulance response to chest pain}

Continued from page 99

five people who have an AMI arrive by ambulance. Data from the USA estimate that only about 50 per cent of people presenting to emergency departments with chest pain arrive by ambulance?

The next step in the "chain of survival" is entry to the emergency care system. This system should enable rapid assessment of patients in the "field" (out of hospital by ambulance staff or in emergency departments) according to simple protocols. Protocols should include rapid transfer to a hospital equipped to manage patients with AMI.

It is not possible to determine from these data the appropriateness of applying the Ambulance Service Chest Pain Protocol. The cases recorded as "chest pain" where glyceryl trinitrate or aspirin were not used may have been judged to be non-ischaemic in origin, such as trauma or other medical conditions, or did not fulfil the criteria that indicate coronary ischaemia.

Approximately one in four patients were given aspirin, though almost two-thirds were given glyceryl trinitrate, for suspected coronary ischaemia. The concern is not so much the failure to give aspirin in itself. The best evidence at present, however, is that it should be commenced within 24 hours of the infarct, and commencement in the ambulance does not necessarily confer additional benefit ${ }^{8}$.

Early recognition of symptoms and entry into the system of emergency care is crucial to ensuring desired health outcomes for people with AMI. In addition, those in whom a thrombolytic is contraindicated and those with unstable angina will benefit from early assessment and treatment to limit disease progression, loss of cardiac function and possible mortality. Nevertheless, delays in delivery of definitive treatment for acute coronary ischaemia have been documented in many studies $9,10,11,12,13,14,15$. The major source of delay seems to be before the decision to seek emergency assistance $^{14}$. Of some concern is the suggestion that people are less likely to seek care expeditiously when they have been diagnosed with coronary heart disease ${ }^{16,17}$.

There are many possible reasons for these delays, including patient and family denial. A decision to seek medical help from the local doctor may significantly increase the time to hospital and definitive care $\mathrm{e}^{13,14}$.

Measures to encourage people (especially those known to be at high risk, such as people with diagnosed $\mathrm{CHD}$ ) to respond appropriately to persisting chest pain and to minimise delays in assessment and definitive management of people in the emergency department have the potential to substantially reduce avoidable mortality and minimise loss of cardiac function.

A systematic approach to management of acute coronary ischaemia is needed to address these issues. The NSW Rural and Metropolitan Critical Care Plans could serve as a model for such an approach. Though these plans have not explicitly addressed coronary ischaemia, they do deal with many generic issues and could provide the architecture for the effective delivery of services for the management of acute coronary ischaemia in NSW.

\section{ACKNOWLEDGMENT}

During the analysis and writing of this paper, SCF has obtained advice from his colleague Marilyn Rob regarding aspects such as presentation, editing and proof reading. We thank Marilyn for her time and effort in doing this for us.

1. Lewis WR, Amsrerdam EA. Utility and Safety of immediate exercise testing of low-risk patients admitted to the hospital for suspected acute myocardial infarction. Am J Cardiol 1994; 74(10):987-90.

2. Fothergill NJ, Hunt MT, Touquet R. Audit of patients with chest pain presenting to an accident and emergency department over a 6-month period. Arch Emerg Med 1993; 10(3):155-60.

3. Ting HH, Lee TH, Soukup JR, Cook EF, Tosteson AN, Brand DA Rouan GW, Goldman L. Impact of physician experience on triage of emergency room patients with acute chest pain at three teaching hospitals. Am J Med 1991; 91(4):401-8.

4. Cummins RO. Emergency medical services and sudden cardiac arrest: "the chain of survival" concept. In Omenn GS, Fielding JE, Lave LB (eds). Annual Review of Public Health 1993; 14: 313-333. 5. Report of the Inquiry into the New South Wales Ambulance Service, July 1982.

6. ORH Review of Ambulance Service Standards Emergency and Urgent Targets - Final Report, Oxford House, UK, 1990.

7. Moss AJ, Wynar B, Goldstein S. Delay in hospitalisation during the acute coronary period. Am J Cardiol 1969; 24:659-665.

8. Antiplatelet Trialist's Collaboration. Collaborative overview of randomised trials of antiplatelet therapy - I: prevention of death myocardial infarction and stroke by prolonged antiplatelet therapy in various categories of patients. BM.J 1994;308:81-106.

9. Weilgosz AT, Nolan RP, Earp JA, Biro E, Weilgosz MB. Reasons for patients' delay in response to symptoms of acute myocardial infarction patients' delay in response to sympto
Can Med Assoc $J$ 1988; 139:853-7.

10. Fields KB. Myocardial infarction and denial. J F c m Pract 1989; 28:157-61.

11. Report of the European Myocardial Infarction Project (EMIP) Subcommittee. Potential time saving with pre-hospital intervention in acute myocardial infarction. Eur Heart $J$ 1988; 9:118-24.

12. Hofgren K, Bondestam E, Johansson G, Jem S, Herlitz J, Holmberg 12. Hofgren $\mathrm{K}$, Bondestam $\mathrm{E}$, Johansson $\mathrm{G}$, Jem $\mathrm{S}$, Herlitz J, Holmber
$\mathrm{S}$. Initial pain course and delay to hospital admission in relation to S. Initial pain course and delay to hospital admission 13. Rawles JM, Haites NE. Patient and general practitioner delays in acute myocardial infarction. BMJ 1988; 296:882-4.

14. Leitch JW, Birbara T, Freedman B, Wilcox I, Harris PJ. Factors influencing the time from onset of chest pain to arrival at hospital. Med J Aust 1989: 150:6-10.

15. Heriot AG, Brecker SJ, Coltart DJ. Delay in presentation after myocardial infarction. J Roy Soc Med 1993; 86:642-644. 16. Tjoe SL, Luria MH. Delays in reaching the cardiac care unit: an analysis. Chest $1972 ; 61: 617-21$.

17. Turi ZG, Stone PH, Muller JE. Implications for acute intervention related to time of hospital arrival in acute myocardial infarction. Am J Cardiol 1986; 58:203-9. 